\title{
microRNA-196a attenuates ischemic brain injury in rats by directly targeting high mobility group A1
}

\author{
JUNAN HU and WEI SHEN

\begin{abstract}
Department of Neurosurgery, Beilun District People's Hospital of Ningbo City, Ningbo, Zhejiang 315800, P.R. China
\end{abstract}
Received May 10, 2016; Accepted November 22, 2018

DOI: $10.3892 /$ etm.2019.7152

\begin{abstract}
Dysfunction of the microRNA (miR) network has been indicated as a major regulator in neurological diseases. However, there is limited understanding regarding the functional significance of miRs in ischemic brain injury. In the present study, miR-196a expression was significantly increased in rat brains and neurons following transient middle cerebral artery occlusion (MCAO) or oxygen-glucose deprivation, respectively. In addition, repression of miR-196a significantly decreased neuron cell apoptosis and the infarct size in rats subjected to MCAO $(\mathrm{P}<0.05)$. Furthermore, miR-196a was indicated to directly target and inhibit high mobility group A1 expression, which indicated a potential role for miR-196a in ischemic brain injury. These findings suggested that miR-196a may be involved in regulating neuronal cell death, thus offering a novel target for the development of therapeutic agents against ischemic brain injury.
\end{abstract}

\section{Introduction}

Ischemic brain injury is caused by insufficient blood flow to the brain, is characterized by oxidative stress, hypoxia, inflammation and glutamate excitotoxicity, which leads to cell apoptosis and death (1). Ischemic stroke has been indicated as a leading cause of death and long-term disability worldwide (2). Neonatal hypoxic-ischemic brain injury or neonatal stroke is a major cause of hypoxic-ischemic encephalopathy and cerebral palsy (3). The advances in therapy for the purpose of decreasing ischemic brain injury have been limited because the pathophysiological mechanisms remain unknown. Therefore, it is of great importance to understand the pathological mechanisms of ischemia-associated cell death in order to develop effective therapies for ischemic brain injury.

Correspondence to: Dr Wei Shen, Department of Neurosurgery, Beilun District People's Hospital of Ningbo City, 1288 East Lu-Shan Road, Ningbo, Zhejiang 315800, P.R. China

E-mail: shenweiningbovip@sina.com

Key words: ischemic brain injury, microRNA-196a, oxygen-glucose deprivation, HMGA1
microRNAs (miRNAs or miRs) are a family of small RNAs ( $\sim 22$ nucleotides) that modify the expression of genes implicated in biological processes, including tumor cell differentiation, proliferation and apoptosis $(4,5)$. Previous studies have indicated that miRNAs are abundantly expressed in the nervous system and have been initially identified as crucial molecular mediators in the regulation of neuronal cell survival (6-8). miR-497 was the first miRNA identified to promote ischemic neuronal death by negatively regulating anti-apoptotic proteins, B-cell lymphoma-2 (Bcl-2) and Bcl-2-like protein 2 (9). miR-210, miR-29b and miR-124 have also been demonstrated to participate in the biological processes concerning the nervous system (10-12).

These findings suggest that several miRNAs may be potential candidates for possible biomarkers or therapeutic targets in stroke. In the present study, the expression of miR-196a was explored during OGD in vitro and MCAO in vivo. The role of miR-196a in ischemic brain injury was investigated.

\section{Materials and methods}

Primary culture of rat cortical neurons. A total of 15 pregnant, female Sprague Dawley rats (mean weight, $245 \pm 10 \mathrm{~g}$ ) were obtain from the SLAC Laboratory Animal Corporation (Shanghai, China). All rats housed at $20-25^{\circ} \mathrm{C}$ with $60 \%$ humidity under $12 \mathrm{~h}$ light:dark cycles with free access to food and water throughout the experiment. After 19-20 days, 115 neonatal rats were born and cerebral cortexes were removed within $24 \mathrm{~h}$ by aseptic decapitation. Then cortexes were placed in D-hanks solution (Gibco; Thermo Fisher Scientific, Inc., Waltham, MA, USA) and sliced into $1-\mathrm{mm}^{3}$ fragments. Subsequently, the cortical pieces were dissociated using $0.25 \%$ trypsin (Sigma-Aldrich; Merck KGaA, Darmstadt, Germany) supplemented with $100 \mathrm{ng} / \mathrm{ml}$ DNase (Roche Diagnostics, Basel, Switzerland) at $37^{\circ} \mathrm{C}$ for 35 min. The digestion was terminated with Dulbecco's modified Eagle's medium (DMEM; Hyclone; Logan, UT, USA) supplemented with $10 \%$ fetal bovine serum (Gibco; Thermo Fisher Scientific, Inc.). Isolated cells were suspended in neurobasal-A medium supplemented with 2\% B27, $1 \%$ glutamine (Gibco; Thermo Fisher Scientific, Inc.), $100 \mathrm{U} / \mathrm{ml}$ penicillin and $100 \mathrm{~g} / \mathrm{ml}$ streptomycin, and seeded into 6-well plates at $5 \times 10^{4}$ cell/well; the plates were precoated with $10 \mu \mathrm{g} / \mathrm{ml}$ poly-D-lysine (Sigma-Aldrich; Merck KGaA). Cells were incubated in a humidified incubator containing $5 \% \mathrm{CO}_{2}$ 
and $95 \% \mathrm{O}_{2}$ at $37^{\circ} \mathrm{C}$ for 7 days. The present study and all animal experiments were approved by the Ethics Committee at Beilun People's Hospital of Ningbo (Ningbo, China).

OGD model. To construct an OGD model, glucose-free Earl's balanced salt solution (EBSS) medium (consisting of $6,800 \mathrm{mg} / \mathrm{l} \mathrm{NaCl}, 400 \mathrm{mg} / \mathrm{l} \mathrm{KCl}, 264 \mathrm{mg} / \mathrm{l} \mathrm{CaCl}{ }_{2}, 200 \mathrm{mg} / 1$ $\mathrm{MgCl}_{2}, 2,200 \mathrm{mg} / \mathrm{l} \mathrm{NaHCO}$ and $140 \mathrm{mg} / 1 \mathrm{NaH}_{2} \mathrm{PO}_{4} \mathrm{mg} / \mathrm{l}$; pH 7.2) was purged with $\mathrm{N}_{2} / \mathrm{CO}_{2}(95 / 5 \%)$ at $37^{\circ} \mathrm{C}$ for $20 \mathrm{~min}$, resulting in an oxygen content of $1 \%$. Cortical neuronal cell cultures were washed three times with glucose-free EBSS medium and incubated at $37^{\circ} \mathrm{C}$ for $4 \mathrm{~h}$ in oxygen-free $\mathrm{N}_{2} / \mathrm{CO}_{2}$ $(95 / 5 \%)$ gas as previously described (13). For reoxygenation, cells were incubated in fresh neurobasal-A-medium supplemented with $2 \%$ B-27 in an atmosphere containing 5\% $\mathrm{CO}_{2}$ at $37^{\circ} \mathrm{C}$ for 12 and $24 \mathrm{~h}$. The control group was incubated in EBSS with $10 \mathrm{mM}$ glucose at $37^{\circ} \mathrm{C}$ in $5 \% \mathrm{CO}_{2}$ for 12 and $24 \mathrm{~h}$.

MCAO rat model. A total of 32 adult male Sprague Dawley rats (age, 10 weeks; mean weight, $289 \pm 10 \mathrm{~g}$ ) were subjected to MCAO from the SLAC Laboratory Animal Corporation. All rats housed at $20-25^{\circ} \mathrm{C}$ with $60 \%$ humidity under $12 \mathrm{~h}$ light:dark cycles with free access to food and water throughout the experiment. Rats were anesthetized using $1 \%$ sodium pentobarbital (40 mg/kg; Shanghai Xitang Company, Shanghai, China). Subsequently, the right common carotid artery, external carotid artery and internal carotid artery were exposed via a midline cervical incision. A piece of $4 / 0$ monofilament nylon suture with a heat-induced rounded tip was inserted through the right internal carotid artery to the base of the middle cerebral artery, which occluded the blood flow to the cortex and striatum. Following $90 \mathrm{~min}$ of MCAO, the rats were allowed to recover for $24 \mathrm{~h}$, as described previously (14). Sham-operated mice underwent the internal carotid artery separation without the suture insertion. Additionally, the rats were randomly separated into four groups: Sham operation, MCAO model, MCAO model treated with antagomiR control (5'-CAGUAC UUUUGUGUAGUACAA-3') and antagomiR-196a (5'-CCC AACAACAUGAAACUACCUA-3'). The antagomiR control and antagomiR-196a (both $80 \mathrm{mg} / \mathrm{kg}$ ) were injected into the caudal vein.

Cell transfection. A total of $2 \times 10^{5}$ cortical neurons were plated in 6-well plates and cultured at $37^{\circ} \mathrm{C}$ for $24 \mathrm{~h}$. miR-196 mimics, miR-196 inhibitor or high mobility group A1 (HMGA1) vectors were transfected using Lipofectamine 2000 (Invitrogen; Thermo Fisher Scientific, Inc.) at a final concentration of $30 \mathrm{mM}$. The sequences for the miRNAs were as follows: NC control (5'-CAGUACUUUUGUGUA GUACAA-3'), miR-155 mimic (5'-UAGGUAGUUUCAUGU UGUUGGG-3'), miR-155 inhibitor (5'-CCCAACAACAUG AAACUACCUA-3'). Neurons were harvested for terminal deoxynucleotidyl-transferase-mediated dUTP nick end labelling (TUNEL) assay, western blot analysis or luciferase activity assay, at the indicated time points.

Infarct volume analysis. A total of $24 \mathrm{~h}$ after reperfusion, the rats were re-anesthetized and sacrificed. Then, the brain tissues of each group were removed, frozen for $30 \mathrm{~min}$ at $-20^{\circ} \mathrm{C}$ and sliced into four coronal sections ( $1 \mathrm{~mm}$ thick). The sections were placed in a $1 \%$ solution of 2,3,5-triphenyltetrazolium chloride (Sigma-Alrich; Merck KGaA) for $10 \mathrm{~min}$ at $37^{\circ} \mathrm{C}$ and fixed in $4 \%$ formalin at $4^{\circ} \mathrm{C}$ for $24 \mathrm{~h}$. The infarct region lacked staining and appeared white, whereas the normal non-infarct tissue appeared red. Images were captured of both sides of each stained coronal slice using a digital camera and infarction was measured using ImageJ Pro 6.0 digital image analysis software (National Institutes of Health, Bethesda, MD, USA). The infarct volume was expressed as a percentage: Total infarct volume of ipsilateral structure/total volume of contralateral structure $\mathrm{x} 100$.

TUNEL assay. Coronal brain tissues were fixed in $10 \%$ paraformaldehyde at $4^{\circ} \mathrm{C}$ for $24 \mathrm{~h}$, dehydrated in ethanol (100, 95, 90, 80 and 70\%; Beijing Solarbio Science \& Technology Co., Ltd., Beijing, China) and embedded in paraffin. The specimens were cut into $4-\mu \mathrm{m}$ thick slices for TUNEL and immunocytochemistry analysis. TUNEL assay was performed according to the manufacturer's instructions of the TdT-mediated dUTP nick end labeling analysis kit (Roche Diagnostics). All cell nuclei were indicated via blue staining and the positive nuclei were indicated via green staining. The number of positive nuclei was determined by manually counting the positively labeled nuclei in five randomly selected fields under a fluorescence microscope (magnification, $\mathrm{x} 400$ ).

Immunocytochemistry. The 4- $\mu$ m thick sections were deparaffinized and rehydrated with ethanol (100, 95 and 75\%), and exposed to the antigen retrieval system (10 mM sodium citrate and $0.05 \%$ Tween $20 ; \mathrm{pH} 6.0$ ) at room temperature for $15 \mathrm{~min}$. Endogenous peroxidase was blocked with $3 \%$ hydrogen peroxide for $15 \mathrm{~min}$ at room temperature. Subsequently, the slides were rinsed in $0.01 \mathrm{~mol} / \mathrm{l} \mathrm{PBS}$ three times and subsequently incubated with the primary mouse polyclonal HMGA1 antibody (1:200; cat. no. ab226112; Abcam, Cambridge, MA, USA) for $1 \mathrm{~h}$ at room temperature. Following washing with PBS twice for $5 \mathrm{~min}$, the slides were incubated with horseradish peroxidase-labeled goat anti-mouse antibody (1:100; cat. no. A0192; Beyotime Institute of Biotechnology, Haimen, China) at room temperature for $1 \mathrm{~h}$. Samples were stained using a 3,3'-diaminobenzidine peroxidase substrate kit (Beyotime Institute of Biotechnology) according to the manufacturer's protocol. Positive nuclei were indicated as brown in color. Slides were observed using a fluorescence microscope (magnification, $\mathrm{x} 400$ ) and the number of positive cells was analyzed by Image $\mathrm{J}$ pro 6.0 .

Reverse transcription-quantitative polymerase chain reaction (RT-qPCR). Total RNA obtained from rats and cells was extracted using TRIzol Reagent (Invitrogen; Thermo Fisher Scientific, Inc.) according to the manufacturer's protocol. A total of $1 \mu \mathrm{g}$ total RNA was reverse-transcribed into cDNA using a TaqMan microRNA Reverse Transcription kit (Applied Biosystems; Thermo Fisher Scientific, Inc.) in a 15- $\mu 1$ total volume according to the manufacturer's protocol. The reactions were incubated for $5 \mathrm{~min}$ at $95^{\circ} \mathrm{C}$, followed by 40 cycles consisting of a $15 \mathrm{sec}$ interval at $95^{\circ} \mathrm{C}$ and $1 \mathrm{~min}$ interval at $60^{\circ} \mathrm{C}$. Expression levels of miR-196a were measured by TaqMan MicroRNA Assay kits (Applied Biosystems; 
Thermo Fisher Scientific, Inc.) in triplicate on an IQ5 real-time PCR system (Bio-Rad Laboratories, Inc., Hercules, CA, USA) and normalized using GAPDH as the internal control. The quantification was performed by the comparative $2^{-\Delta \Delta \mathrm{Cq}}$ method (15). The primer sequences $\left(5^{\prime} \rightarrow 3^{\prime}\right)$ were: HMGA1, forward: TTACCGAGTACCCCACGCTA, reverse: AGG CTGGGACAAATACTGGC; GAPDH, forward: GATGGT GAAGGTCGGTGTGA, reverse: TGAACTTGCCGTGGG TAGAG; miR-196a RT-primer: CTCAACTGGTGTCGTGGA GTCGGCAATTCAGTTGAGCCCAACAA, forward: ACA CTCCAGCTGGGTAGGTAGTTTCATGTT, reverse: TGG TGTCGTGGAGTCG.

Western blot analysis. Total proteins from cells were extracted with radioimmunoprecipitation assay lysis buffer (Beyotime Institute of Biotechnology) and protein concentration was tested using the BCA assay (Beijing Solarbio Science \& Technology Co., Ltd.). A total of $30 \mu \mathrm{g}$ protein were loaded and separated using 10\% Tris-glycine SDS-PAGE, and transferred to polyvinylidene fluoride membranes (EMD Millipore, Billerica, MA, USA) using the electrophoretic transfer system (Bio-Rad Laboratories, Inc.). The membranes were blocked with $5 \%$ non-fat dry milk in Tris- $\mathrm{HCl}$ buffer saline $(\mathrm{pH} 7.4)$ containing $0.1 \%$ Tween 20 for $1 \mathrm{~h}$ at room temperature and incubated with primary antibodies against HMGA1 and GAPDH (both 1:1,000; cat. no. ab181602; Abcam) overnight at $4^{\circ} \mathrm{C}$. Subsequently, the membranes were incubated with horseradish peroxidase-conjugated secondary antibodies $(1: 2,500$; cat. no. A0192) at room temperature for $2 \mathrm{~h}$ followed by band detection with a Super Signal Enhanced Chemiluminescence Kit (Pierce; Thermo Fisher Scientific, Inc.). Quantification of the bands was performed using densitometric analysis and the ImageJ Pro 6.0 analysis system (National Institutes of Health).

Bioinformatics analysis. To predict the target genes of miR-196a, a series of bioinformatics analyses were used. The authors predicted the target genes for miR-196a with miRbase (http://www.mirbase.org/), TargetScan (http://www.targetscan. org/) and miRDB (mirdb.org/miRDB/). The predicted targets included HMGA1.

Luciferase assay. Cortical neuronal cells were plated at a density of $4 \times 10^{3}$ cells/well in 96-well plates and cultured at $37^{\circ} \mathrm{C}$ for $24 \mathrm{~h}$. Cells were co-transfected with $40 \mathrm{ng}$ recombinant wild-type (WT) or mutated (MUT) luciferase vectors [pGL3-HMGA1-3'-untranslated region (UTR)-WT or pGL3-HMGA1-3'-UTR-Mut], pRL-TK vectors and miR-196a mimics or a negative control (NC) mimics (Guangzhou RiboBio Co., Ltd., Guangzhou, China) at a final concentration of 45 nM using Lipofectamine 2000 (Invitrogen; Thermo Fisher Scientific, Inc.) according to the manufacturer's instructions. Luciferase assays were performed with a dual luciferase reporter assay system (Promega Corporation, Madison, WI, USA) $48 \mathrm{~h}$ following transfection. Renilla luciferase activity was normalized to that of Firefly luciferase.

Statistical analysis. All data were expressed as the mean \pm standard deviation of at least three separate experiments. Difference between two groups was compared by Student's t-test. Difference among multiple groups was analyzed by one-way analysis of variance followed by a post-hoc Student-Neuman-Keuls test. $\mathrm{P}<0.05$ was considered to indicate a statistically significant difference.

\section{Results}

Expression of miR-196a and HMGAl was changed in cortical neurons subjected to OGD. To investigate the role of miR-196a and HMGA1 in ischemic brain injury, an OGD model of cortical neurons was established. RT-qPCR was performed to evaluate the gene expression levels (Fig. 1). As Fig. 1B demonstrated, OGD induced apoptosis and the difference between the control and OGD groups at 12 and $24 \mathrm{~h}$ was significant $(\mathrm{P}<0.05$; Fig. 1A). Notably, the miR-196a expression was elevated significantly following OGD at 12 and $24 \mathrm{~h}$ compared with the control $(\mathrm{P}<0.05$; Fig. 1C). Conversely, the mRNA and protein expression levels of HMGA1 were significantly downregulated after 12 and $24 \mathrm{~h}$ of OGD compared with the control $(\mathrm{P}<0.05$; Fig. 1D and $\mathrm{E})$.

miR-196a reduces the neuronal rate of apoptosis and infarction following ischemic brain injury in vivo. A rat MCAO model was constructed to further explore the effect of miR-196a on ischemic brain injury and its mechanism (Fig. 2). The expression of miR-196a was determined to assess the effects of antagomiR-196a. As presented in Fig. 2A, miR-196a expression in MCAO was significantly higher than the sham group $(\mathrm{P}<0.01)$, whereas antagomiR-196a injection significantly decreased the miR-196a expression compared with the antagomiR control group $(\mathrm{P}<0.05)$. The infarct volume and cortical neuron apoptosis rate was increased in the MCAO group compared with the sham and this difference was indicated as statistically significant $(\mathrm{P}<0.05$; Fig. 2B-E), which indicated the successful establishment of the in vivo model. As expected, knockout of miR-196a by intracerebroventricular injection of antagomiR-196a resulted in a significant decrease in miR-196a expression compared with the MCAO group $(\mathrm{P}<0.05$; Fig. 2A) and significantly improved the recovery of brain function as the infarct volume and the apoptosis rate were significantly decreased compared with the MCAO groups $(\mathrm{P}<0.05$; Fig. 2B-E). Furthermore, TUNEL staining indicated that the proportion of apoptotic cells was decreased in miR-196a antagomiR-injected rat brains compared with the antagomir control (Fig. 2D) and the difference was statistically significant (Fig. 2E). Considering the abnormal expression of HMGA1 in cells undergoing OGD (Fig. 1E), the expression of HMGA1 using immunocytochemistry in rat brains subjected to MCAO was evaluated. Consistent with the previous study, the expression of HMGA1 was markedly downregulated in the MCAO group compared with that in the sham group and antagomir-196a injection significantly enhanced the HMGA1 expression to sham group ( $\mathrm{P}<0.05$; Fig. $2 \mathrm{~F}$ and $\mathrm{G}$, respectively).

miR-196a directly targets HMGA1 and inhibits its expression. To identify the target genes of miR-196a in regulating ischemic brain injury, candidate genes were investigated using miRBase, TargetScan 5.1 and micoRNA databases. HMGA1 was selected as a potential candidate gene due to its abnormal expression in cortical neuronal cells subjected to OGD and in rat brains following MCAO. miR-196a mediated HMGA1 
A

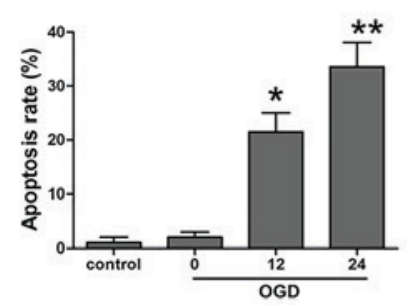

D

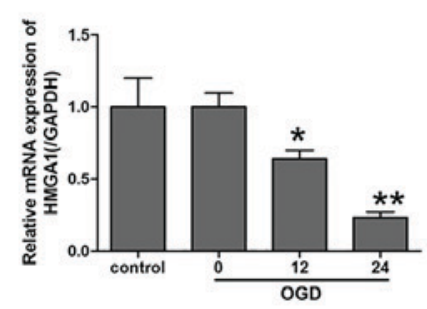

B

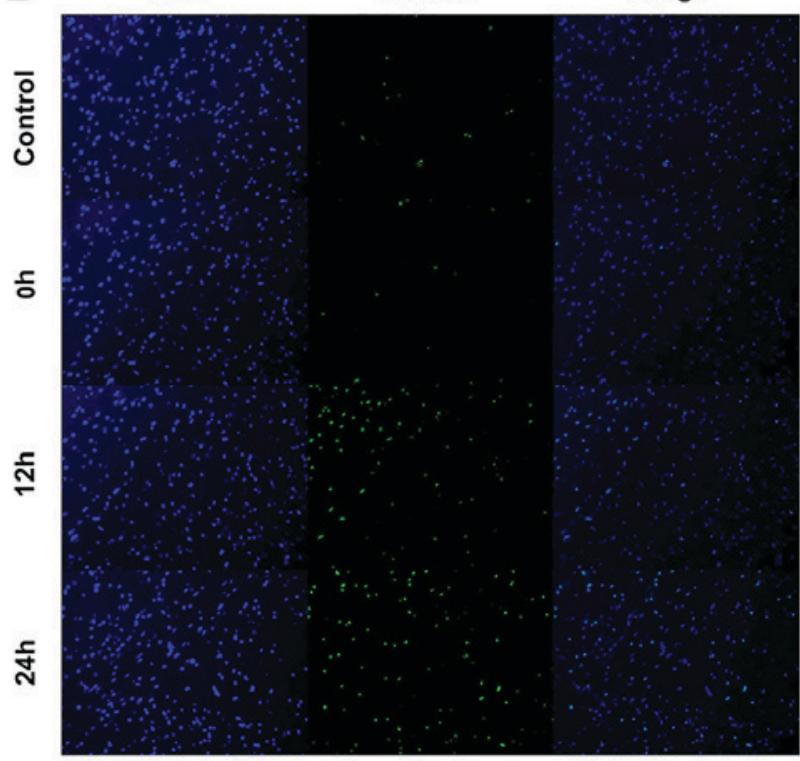

C
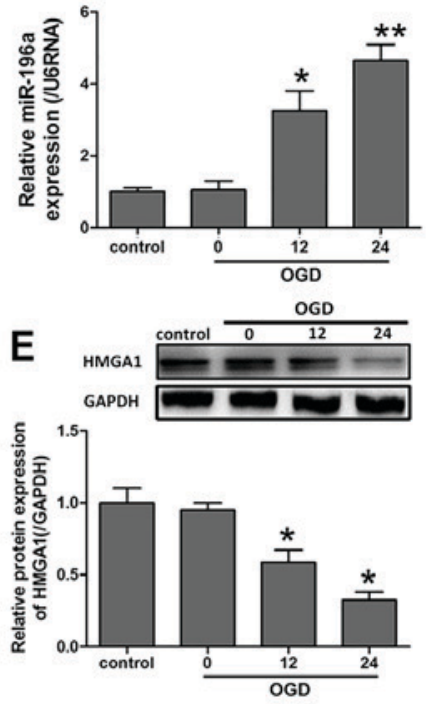

Figure 1. Expression of miR-196a and HMGA1 in cortical neurons subjected to OGD. (A and B) TUNEL was used to evaluate the apoptosis of cortical neurons following OGD (magnification, x100). Blue fluorescence indicates nuclei and green fluorescence indicates apoptotic cells. Reverse transcription-quantitative polymerase chain reaction was used to determine the expression of (C) miR-196a and (D) HMGA1. (E) Protein expression levels of HMGA1 were determined using western blot analysis. Data are expressed as the mean \pm standard deviation $(\mathrm{n}=8)$. ${ }^{*} \mathrm{P}<0.05$ and ${ }^{* *} \mathrm{P}<0.01$ vs. control. HMGA1, high mobility group A1; ODG, oxygen-glucose deprivation; miR-196a, microRNA-196a; TUNEL, terminal deoxynucleotidyl-transferase-mediated dUTP nick end labeling; DAPI, 4',6-diamidino-2-phenylindole.

expression by directly binding to the 3'UTR of HMGA1 in cortical neuronal cells (Fig. 3A). Luciferase assay was performed to indicate whether miR-196a targeted HMGA1. Co-transfection of miR-196a mimics significantly inhibited the luciferase activity produced by the reporter construct that contained the wild-type 3'UTR segment of HMGA1 $(\mathrm{P}<0.05)$, whereas no significant effect was observed with the construct containing the mutated segment of HMGA1 3'UTR (Fig. 3B). RT-qPCR demonstrated that miR-196a expression was significantly increased in the miR-196a mimics group, but was reduced in miR-196a inhibitor group when compared with the OGD group $(\mathrm{P}<0.05$; Fig. $3 \mathrm{C})$. In addition, western blot analysis was applied to observe the protein expression levels of HMGA1. The results revealed that transfection of miR-196a mimics significantly decreased HMGA1 in OGD-induced cells, whereas miR-196a inhibitor increased HMGA1 compared with the OGD group $(\mathrm{P}<0.05$; Fig. 3D).

HMGAl overexpression suppresses cell apoptosis in cortical neurons subjected to OGD. To directly determine the effect of HMGA1 on OGD-induced neuronal damage, neuronal cells were transfected with pcDNA3.1/HMGA1 vector and the TUNEL assay was performed to evaluate the apoptosis of cortical neurons under different treatments (Fig. 4A). Compared with the control, HMGA1 overexpression significantly suppressed the apoptosis induced by OGD $(\mathrm{P}=0.043$; Fig. 4B).

HMGA1 reverses the induction of apoptosis by $m i R-196 a$. In accordance with the findings in the in vivo study, miR-196a overexpression was indicated to promote the apoptosis in OGD neurons. To explore whether miR-196a targets HMGA1, neuronal cells were co-transfected with HMGA1 vector and
miR-196a mimics. As expected, HMGA1 overexpression significantly reversed the induction of apoptosis by miR-196a $(\mathrm{P}<0.05$; Fig 4B).

\section{Discussion}

miRNA binding is mediated by argonaute proteins within the RNA-induced silencing complex, which is dependent on the degree of sequence complementarity and leads to either cleavage of the target mRNA or a reduction in its translational efficiency (16). A subset of miRNAs are abundantly expressed in the human brain (17) and have critical roles in the pathophysiology of brain seizures, ischemia and trauma $(18,19)$.

miR-196a was initially identified as an oncogene that was associated with apoptosis, invasion and proliferation (20). Increasing evidence has suggested the aberrant expression of miR-196a is a frequent event in various cancers, including head and neck squamous cell carcinomas, laryngeal cancer, pancreatic cancer and gastric cancer (21-24). In addition, miR-196a is a putative diagnostic biomarker or therapeutic target for various human diseases, including adrenomyeloneuropathy, chronic hepatitis and Huntington's Disease (25-27). To date, limited understanding exists regarding the association between miR-196a and ischemic brain injury. In the present study, miR-196a expression was significantly increased during OGD in vitro and $\mathrm{MCAO}$ in vivo. To determine whether miR-196a has a role in ischemic brain injury, its expression was downregulated and the effects explored. The results demonstrated that knockdown of endogenous miR-196a expression significantly reduced brain infarct size and protected against ischemic-induced brain cell apoptosis. To identify the target genes of miR-196a in regulating cell 
A

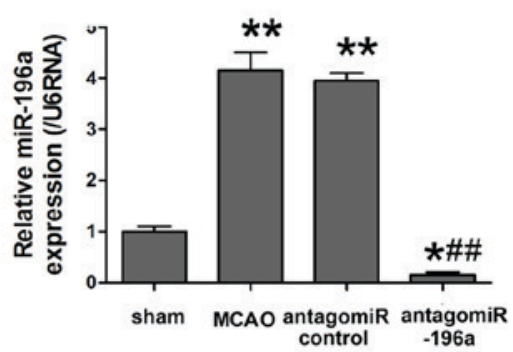

B
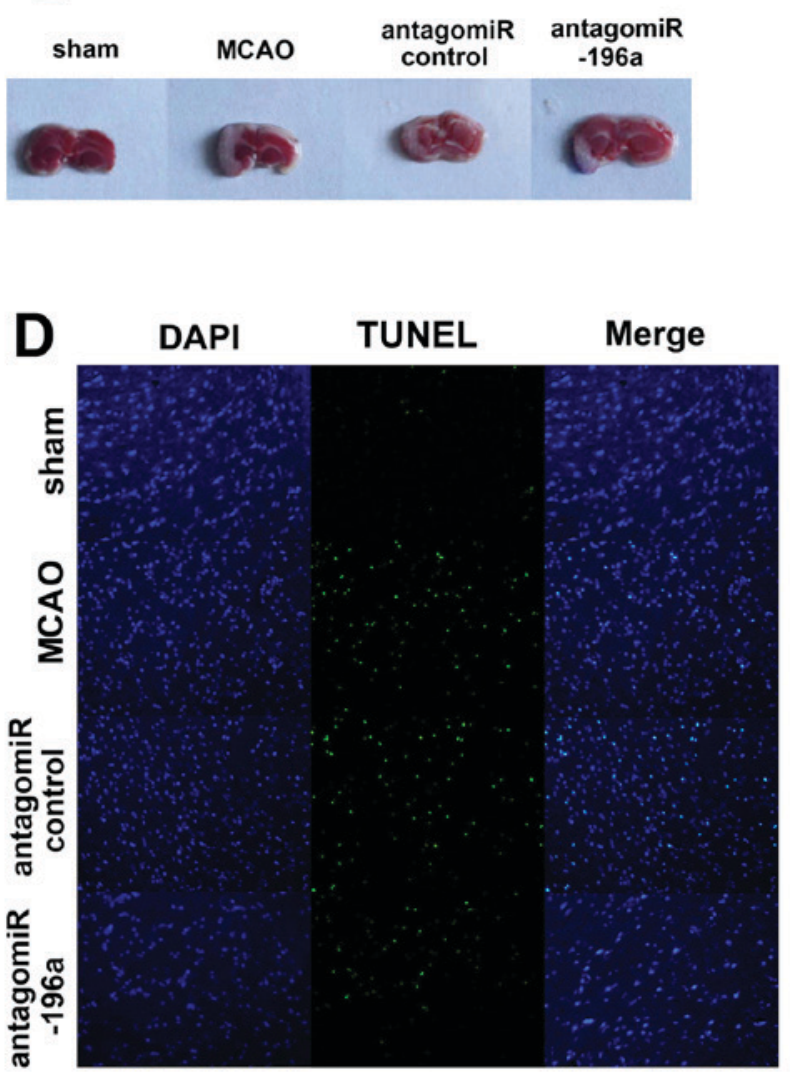

$\mathbf{F}$

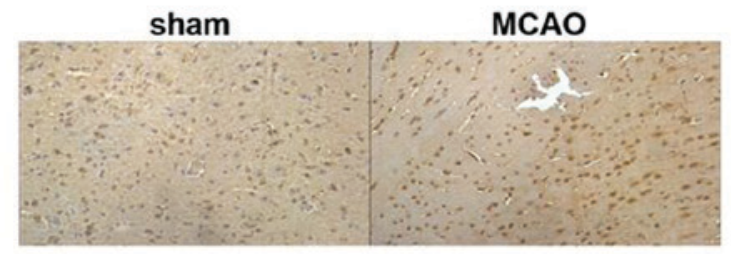

antagomiR control

antagomiR-196a

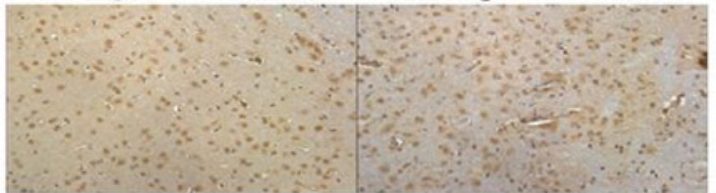

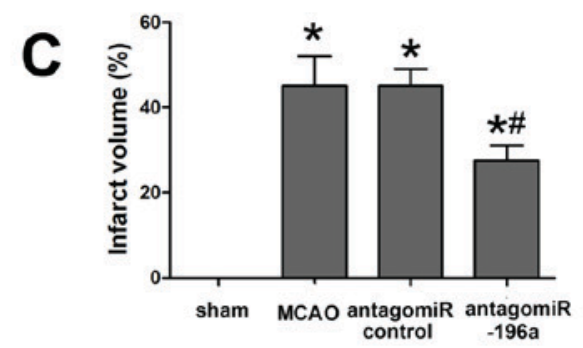

$\mathbf{E}$

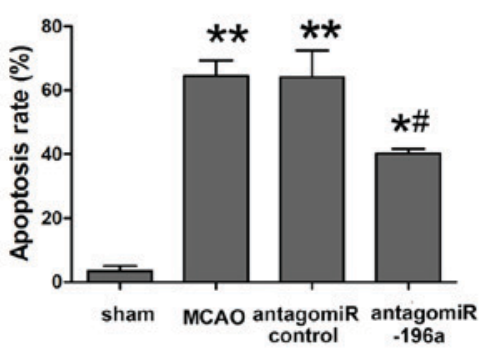

G

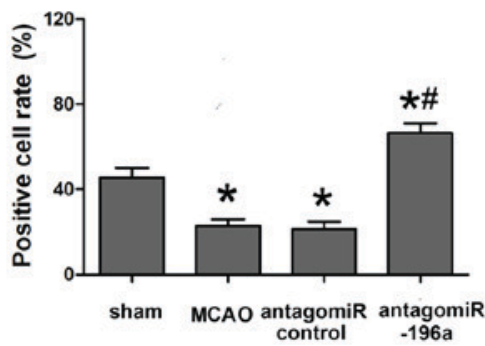

Figure 2. miR-196a reduced neuronal apoptosis and infarction following ischemic brain injury in vivo. (A) Rats were pretreated with a miR-196a antagomir, antagomir control and subsequently subjected to $1.5 \mathrm{~h} \mathrm{MCAO}$ and $24 \mathrm{~h}$ reperfusion. Reverse transcription-quantitative polymerase chain reaction was used to determine the expression of miR-196a in rat brain tissues. (B) Coronal sections exhibiting the different treatments were stained with $1 \% 2,3,5$-triphenyltetrazolium chloride and were evaluated. The infarct region lacked staining and appeared white, whereas the normal non-infarct tissue appeared red. (C) The infarct volume was determined and (D and E) TUNEL assay was performed to evaluate the apoptosis of brain tissue following MCAO (magnification, x100). Blue fluorescence indicates nuclei and green fluorescence indicates apoptotic cells. (F and G) Immunocytochemistry was used to evaluate the HMGA1 expression in brain tissue. Positive nuclei were indicated as brown in color. Data are expressed as the mean \pm standard deviation $(\mathrm{n}=8)$. ${ }^{*} \mathrm{P}<0.05$ and ${ }^{* * *} \mathrm{P}<0.01 \mathrm{vs}$. the sham group; " $\mathrm{P}<0.05$ vs. the MCAO group. HMGA1, high mobility group A1; miR-196a, microRNA-196a; MCAO, middle cerebral artery occlusion; TUNEL, terminal deoxynucleotidyl-transferase-mediated dUTP nick end labeling. 


\section{A HMGA1-3'UtR WT 5 ' ...UUCCUCUgUUCACAAACUACCUC... \\ rno-miR-196a 3' GGGUUGUUGUACUUUGAUGGAU \\ HMGA1-3'UTR MUT $\quad 5^{\prime}$ '...UUCCUCUGUUCACAAAGUUCGUC...}

B
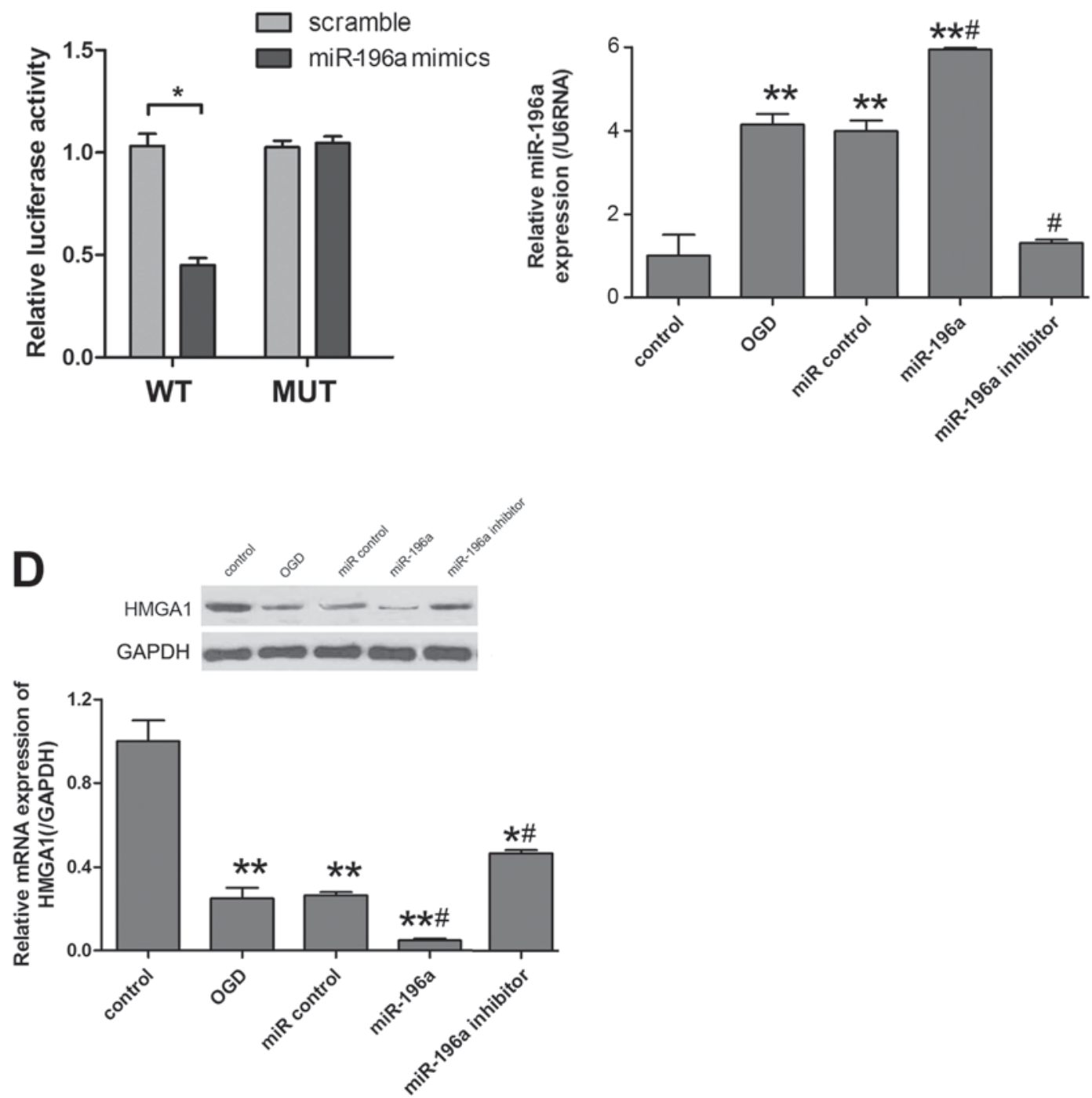

Figure 3. miR-196a directly targeted HMGA1 and inhibited its expression. (A and B) The 3'UTR reporter assay was performed and miR-196a was overexpressed in coronal neurons. pGL3-HMGA1-3'-UTR-WT or pGL3-HMGA1-3'-UTR-Mut was co-transfected with pRL-TK using Lipofectamine 2000. "P<0.05 as indicated. (C) Reverse transcription-quantitative polymerase chain reaction analyses of miR-196a level in different treatment groups. (D) Western blot analysis was used to access the protein expression of neuron cells exposed to different treatment. Data are expressed as the mean \pm standard deviation ( $\mathrm{n}=8$ ). ${ }^{*} \mathrm{P}<0.05$ and ${ }^{* *} \mathrm{P}<0.01$ vs. control; ${ }^{*} \mathrm{P}<0.05$ vs. the OGD group. WT, wild-type; MUT, mutant; UTR, untranslated region; HMGA1, high mobility group A1; miR-196a, microRNA-196a; OGD, oxygen-glucose deprivation; 3'UTR, 3'untranslated region.

apoptosis, miRBase and TargetScan 5.1 were used to search for candidate genes. Due to the low expression of HMGA1 in the current study, HMGA1 was selected as a potential target gene for miR-196a. However, whether miR-196a directly targets HMGA1 required further elucidation. Luciferase assay results indicated that miR-196a significantly repressed luciferase activity in the cortical neuron cells transiently transfected with wide-type HMGA1 3'UTR compared with cells transfected with mutated HMGA1 3'UTR, which suggested that HMGA1 may be a direct target of miR-196a. 
A

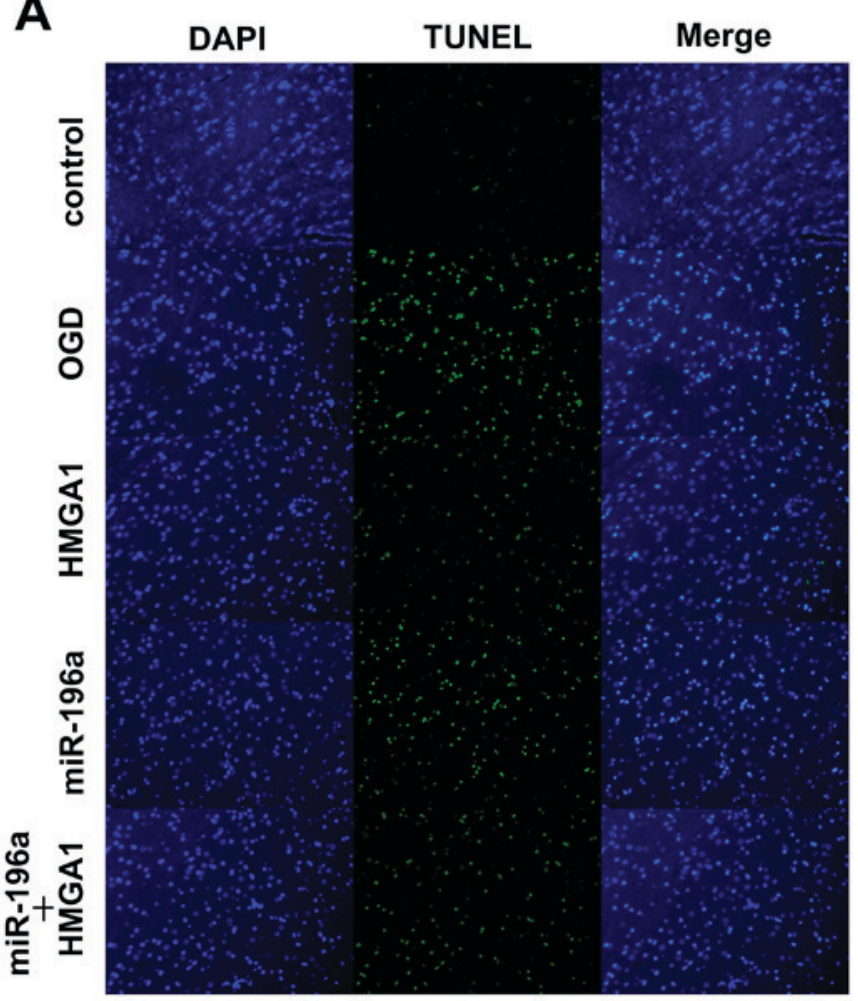

B

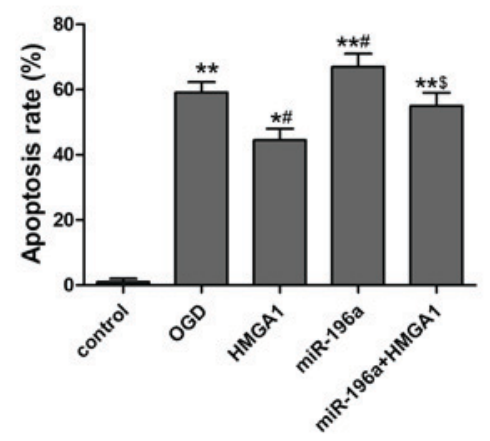

Figure 4. HMGA1 reversed the induction of apoptosis by miR-196a (A and B) TUNEL assay was used to evaluate the apoptosis of cortical neurons exposed to different treatments, (magnification, x100). Blue fluorescence indicates nuclei and green fluorescence indicates apoptotic cells. Data are expressed as the mean \pm standard deviation $(n=8)$. ${ }^{*} \mathrm{P}<0.05$ and ${ }^{* * *} \mathrm{P}<0.01$ vs. control; ${ }^{\mathrm{P}}<0.05$ vs. the OGD group; ${ }^{\mathrm{P}} \mathrm{P}<0.05$ vs. the miR-196a group. HMGA1, high mobility group A1; miR-196a, microRNA-196a; OGD, oxygen-glucose deprivation; TUNEL, terminal deoxynucleotidyl-transferase-mediated dUTP nick end labeling.

In addition, western blot analysis revealed that knockdown of miR-196a significantly increased the protein expression of HMGA1. The present study indicated that HMGA1 may be a physiological target of miR-196a and regulate its expression at the transcriptional level.

HMGA1 proteins are non-histone proteins that regulate chromatin structure and gene expression during embryogenesis, tumourigenesis and immune responses $(28,29)$. HMGA1 is considered as a key hub for several oncogenic pathways, including the Wnt/ß-catenin, Notch pathways and Ras/extracellular-related kinase signaling $(30,31)$. Previous results have indicated that HMGA1 represses p53 apoptotic activity by promoting the cytoplasmic relocalization of the p53 proapoptotic activator homeodomain interacting protein kinase 2 (32). Furthermore, the anti-apoptotic effect of HMGA1 has also been associated with the deregulation of Bcl-2 (33). In addition, ischemic brain injury has been suggested to be associated with widespread molecular and biochemical changes, inflammatory responses, oxidative stress, free radical production and neuronal apoptosis (34-36). Notably, neuronal apoptosis is the prominent cause of cell death in secondary brain damage, which greatly affects the functional outcome of ischemic injury (37). It was hypothesized that miR-196a targeted HMGA1 and regulated neuronal apoptosis following OGD and MCAO. However, the underlying mechanism requires further exploration and further studies are required to explore whether HMGA1 regulates $\mathrm{p} 53$ or $\mathrm{Bcl}-2$.

In conclusion, the present study demonstrated that miR-196a was induced by ischemia and may contribute to the pathogenesis of ischemic brain injury by directly targeting HMGA1. Accordingly, the inhibition of miR-196a may become a potential therapeutic option for ischemia-associated brain damage. However, further studies using neuron-specific miR-196a are warranted.

\section{Acknowledgements}

Not applicable.

\section{Funding}

No funding was received.

\section{Availability of data and materials}

The datasets used and/or analyzed during the present study are available from the corresponding author on reasonable request.

\section{Authors' contributions}

JH designed the study and collected the data. WS analyzed the data and drafted the manuscript. All authors read and approved the final version of the manuscript.

\section{Ethics approval and consent to participate}

The present study and all animal experiments were approved by the Ethics Committee at Beilun People's Hospital of Ningbo (Ningbo, China).

\section{Patient consent for publication}

Not applicable.

\section{Competing interests}

The authors declare that they have no competing interests.

\section{References}

1. Lo EH, Dalkara T and Moskowitz MA: Mechanisms, challenges and opportunities in stroke. Nat Rev Neurosci 4: 399-415, 2003.

2. Elkind MS: Outcomes after stroke: Risk of recurrent ischemic stroke and other events. Am J Med 122 (4 Suppl 2): S7-S13, 2009. 
3. Nelson KB: Perinatal ischemic stroke. Stroke 38 (2 Suppl): S742-S745, 2007.

4. Bartel DP: MicroRNAs: Genomics, biogenesis, mechanism, and function. Cell 116: 281-297, 2004.

5. Bartel DP: MicroRNAs: Target recognition and regulatory functions. Cell 136: 215-233, 2009.

6. Kosik KS: The neuronal microRNA system. Nat Rev Neurosci 7 : 911-920, 2006.

7. Kole AJ, Swahari V, Hammond SM and Deshmukh M: miR-29b is activated during neuronal maturation and targets $\mathrm{BH} 3$-only genes to restrict apoptosis. Genes Dev 25: 125-130, 2011.

8. Yadav S, Pandey A, Shukla A, Talwelkar SS, Kumar A, Pant AB and Parmar D: MiR-497 and miR-302b regulate ethanol induced neuronal cell death through BCL2 and cyclin D2. J Biol Chem 286: 37347-3757, 2011.

9. Yin KJ, Deng Z, Huang H, Hamblin M, Xie C, Zhang J and Chen YE: miR-497 regulates neuronal death in mouse brain after transient focal cerebral ischemia. Neurobiol Dis 38: 17-26, 2010.

10. Jiang Y, Li L, Tan X, Liu B, Zhang Y and Li C: miR-210 mediates vagus nerve stimulation-induced antioxidant stress and anti-apoptosis reactions following cerebral ischemia/reperfusion injury in rats. J Neurochem 134: 173-181, 2015.

11. Shi G, Liu Y, Liu T, Yan W, Liu X, Wang Y, Shi J and Jia L: Upregulated miR-29b promotes neuronal cell death by inhibiting Bcl2L2 after ischemic brain injury. Exp Brain Res 216: 225-230, 2012.

12. Clark AM, Goldstein LD, Tevlin M, Tavaré S, Shaham S and Miska EA: The microRNA miR-124 controls gene expression in the sensory nervous system of Caenorhabditis elegans. Nucleic Acids Res 38: 3780-3793, 2010.

13. Wang Q, Gong Q, Wu Q and Shi J: Neuroprotective effects of Dendrobium alkaloids on rat cortical neurons injured by oxygen-glucose deprivation and reperfusion. Phytomedicine 17: $108-115,2010$

14. Shi GD, OuYang YP, Shi JG, Liu Y, Yuan W and Jia LS: PTEN deletion prevents ischemic brain injury by activating the mTOR signaling pathway. Biochem Biophys Res Commun 404: 941-945, 2011.

15. Livak KJ and Schmittgen TD: Analysis of relative gene expression data using real-time quantitative PCR and the 2(-Delta Delta C(T)) method. Methods 25: 402-408, 2001.

16. Peng B, Chen Y and Leong KW: MicroRNA delivery for regenerative medicine. Adv Drug Deliv Rev 88: 108-122, 2015.

17. Bak M, Silahtaroglu A, Møller M, Christensen M, Rath MF Skryabin B, Tommerup N and Kauppinen S: MicroRNA expression in the adult mouse central nervous system. RNA 14 432-444, 2008

18. Liu DZ, Tian Y, Ander BP, Xu H, Stamova BS, Zhan X, Turner RJ, Jickling G and Sharp FR: Brain and blood microRNA expression profiling of ischemic stroke, intracerebral hemorrhage, and kainate seizures. J Cereb Blood Flow Metab 30: 92-101, 2010.

19. Ziu M, Fletcher L, Rana S, Jimenez DF and Digicaylioglu M: Temporal differences in microRNA expression patterns in astrocytes and neurons after ischemic injury. PLoS One 6: e14724, 2011.

20. Schimanski CC, Frerichs K, Rahman F, Berger M, Lang H, Galle PR, Moehler M and Gockel I: High miR-196a levels promote the oncogenic phenotype of colorectal cancer cells. World J Gastroenterol 15: 2089-2096, 2009.

21. Tsai KW, Liao YL, Wu CW, Hu LY, Li SC, Chan WC, Ho MR, Lai CH, Kao HW, Fang WL, et al: Aberrant expression of miR-196a in gastric cancers and correlation with recurrence. Genes Chromosomes Cancer 51: 394-401, 2012

22. Liu M, Du Y, Gao J, Liu J, Kong X, Gong Y, Li Z, Wu H and Chen $\mathrm{H}$ : Aberrant expression miR-196a is associated with abnormal apoptosis, invasion, and proliferation of pancreatic cancer cells. Pancreas 42: 1169-1181, 2013.
23. Saito K, Inagaki K, Kamimoto T, Ito Y, Sugita T, Nakajo S, Hirasawa A, Iwamaru A, Ishikura $\mathrm{T}$, Hanaoka $\mathrm{H}$, et al: MicroRNA-196a is a putative diagnostic biomarker and therapeutic target for laryngeal cancer. PLoS One 8: e71480, 2013.

24. Severino P, Brüggemann H, Andreghetto FM, Camps C, Klingbeil Mde F, de Pereira WO, Soares RM, Moyses R, Wünsch-Filho V, Mathor MB, et al: MicroRNA expression profile in head and neck cancer: HOX-cluster embedded microRNA-196a and microRNA-10b dysregulation implicated in cell proliferation. BMC Cancer 13: 533, 2013.

25. Shah N and Singh I: MicroRNA profiling identifies miR-196a as differentially expressed in childhood adrenoleukodystrophy and adult adrenomyeloneuropathy. Mol Neurobiol 54: 1392-1403, 2017.

26. Hong XL, Cao H, Zhao F, Pan XF, Zhang K, Xu QH, Zhao ZX and Li G: MiR-196a-2 gene polymorphism and the antiviral therapy of chronic hepatitis C. Zhonghua Shi Yan He Lin Chuang Bing Du Xue Za Zhi 24: 470-472, 2010 (In Chinese).

27. Fu MH, Li CL, Lin HL, Tsai SJ, Lai YY, Chang YF, Cheng PH, Chen CM and Yang SH: The potential regulatory mechanisms of miR-196a in huntington's disease through bioinformatic analyses. PLoS One 10: e0137637, 2015.

28. Shah SN and Resar L: High mobility group A1 and cancer: Potential biomarker and therapeutic target. Histol Histopathol 27: 567-579, 2012.

29. Moussavi Nik SH, Newman M and Lardelli M: The response of HMGA1 to changes in oxygen availability is evolutionarily conserved. Exp Cell Res 317: 1503-1512, 2011.

30. Xing J, Cao G and Fu C: HMGA1 interacts with $\beta$-catenin to positively regulate $\mathrm{Wnt} / \beta$-catenin signaling in colorectal cancer cells. Pathol Oncol Res 20: 847-851, 2014

31. Treff NR, Pouchnik D, Dement GA, Britt RL and Reeves R: High-mobility group Ala protein regulates Ras/ERK signaling in MCF-7 human breast cancer cells. Oncogene 23: 777-785, 2004.

32. Pierantoni GM, Rinaldo C, Mottolese M, Di Benedetto A, Esposito F, Soddu S and Fusco A: High-mobility group A1 inhibits 533 by cytoplasmic relocalization of its proapoptotic activator HIPK2. J Clin Invest 117: 693-702, 2007.

33. Tang D, Kang R, Cheh CW, Livesey KM, Liang X, Schapiro NE, Benschop R, Sparvero LJ, Amoscato AA, Tracey KJ, et al: HMGB1 release and redox regulates autophagy and apoptosis in cancer cells. Oncogene 29: 5299-5310, 2010.

34. Keller JN, Kindy MS, Holtsberg FW, St Clair DK, Yen HC, Germeyer A, Steiner SM, Bruce-Keller AJ, Hutchins JB and Mattson MP: Mitochondrial manganese superoxide dismutase prevents neural apoptosis and reduces ischemic brain injury: Suppression of peroxynitrite production, lipid peroxidation, and mitochondrial dysfunction. J Neurosci 18: 687-697, 1998.

35. Chan PH: Reactive oxygen radicals in signaling and damage in the ischemic brain. J Cereb Blood Flow Metab 21: 2-14, 2001

36. Kriz J: Inflammation in ischemic brain injury: Timing is important. Crit Rev Neurobiol 18: 145-157, 2006.

37. Fluiter K, Opperhuizen AL, Morgan BP, Baas F and Ramaglia V: Inhibition of the membrane attack complex of the complement system reduces secondary neuroaxonal loss and promotes neurologic recovery after traumatic brain injury in mice. J Immunol 192: 2339-2348, 2014.

(i) (5) This work is licensed under a Creative Commons Attribution-NonCommercial-NoDerivatives 4.0 International (CC BY-NC-ND 4.0) License. 